\title{
A New Information Sharing Mechanism Based On Distributed Information Storage Model
}

\author{
Xiaoxuan $\mathrm{Ma}^{1}$, Yiping Huang ${ }^{2}$ and Junyan $\mathrm{Yi}^{3}$ \\ ${ }^{1}$ School of Electrical and Information Engineering, Beijing University of Civil \\ Engineering and Architecture, Beijing 10044, China \\ ${ }^{2}$ School of Electrical and Information Engineering, Beijing University of Civil \\ Engineering and Architecture, Beijing 10044, China \\ ${ }^{3}$ School of Electrical and Information Engineering, Beijing University of Civil \\ Engineering and Architecture, Beijing 10044, China \\ Imaxiaoxuan@bucea.edu.cn, ${ }^{2}$ huangyiping@bucea.edu.cn, \\ 3yijunyan@bucea.edu.cn
}

\begin{abstract}
In WWW, due to the distribution of information resources, how to manage and maintain distributed information resources in different nodes is one of the most important problems in the current research. On the basis of analyzing the scalability, efficiency and effectiveness of data exchange, the paper designed a distributed storage model, discussed the organization of the registry information, put forward a dynamically register and update mechanism, designed a distributed information search algorithm. Experimental results show that the biased flooding search algorithm based on index can not only reduce the message load but also can improve the search hit rate.
\end{abstract}

Keywords: Storage Model, Information Sharing; Distributed Computing

\section{Introduction}

In wide area network, information resources are distributed in different nodes for management and maintenance. Data service center's main goal is to provide information sharing service to users, so that the resource information is better described which is beneficial to resource distribution and discovery, provide fast downloads for resources, etc [2-3]. At the same time, data sharing and exchange is one of the core problems in wide area network information system. In order to meet these goals, the key problems of data service in the data exchange, such as scalability, efficiency and the accuracy and validity are analyzed, and possible solution strategies are put forward.

In wide area network, regardless of e-government or e-commerce, there are a huge amount of information and many different kinds of information. Using a single directory server to manage the share data with all the relevant information will be a single point of failure and too large server load. If undertake unity management it will lead to the increasing cost and poor flexibility. Therefore a hybrid structure model is considered to improve the extensibility.

Data service center's main purpose is to optimize the process of data storage, improve storage efficiency. Therefore, a highly efficient way of information resources organization and the corresponding resource search algorithm are required to improve search efficiency and accuracy.

Because information resources are dynamic, it's required to design a information resources dynamic registration and update mechanism to ensure effectiveness and real-time of resources, at the same time to reduce the load caused by information update. 
Based on the analysis of the key problems and possible solving strategy, combined with the particularity of data exchange under the wan, on the basis of the Web services, P2P technologies, a distributed information storage model based on hybrid structure is proposed $[1,4,5]$. On the basis of the distributed information storage model, tree structure is used as a storage model to group the super node layer registry, which benefits the publication and discovery of resources, improve the extensibility and flexibility. In accordance with the above design idea, the solution for data service center efficiency, accuracy and effectiveness of the solution is proposed. Firstly, the distributed information storage model is designed to solve the scalability problems; Secondly, a information resources dynamic registration dynamic update mechanism is proposed for information resources register and update to ensure the validity of the information resources; Thirdly, is suitable for the model of distributed information resources search algorithm, improves the search efficiency and accuracy of system.

This paper is organized as follows: Section 2 analyzes the distributed information resource storage model and its composition; Section 3 introduces the organization of the registry and its entry to and exit mechanism; Section 4 discusses the information resources dynamic registration and updating method; Section 5 presents a distributed information resource search algorithm, and has carried on the simulation experiment; The work is summarized in Section 6.

\section{Distributed Information Resource Storage Model}

The centralized storage model is a common way of information resource discovery and Shared resources, such as web search engines [6]. In this kind of storage model, all information resources are stored in a single server. The advantage of centralized storage is relatively simple and easy to unified management. But in E-Commerce and E-Government applications, all resources information stored in a server will have the following disadvantages: firstly, the server overload will affect the access efficiency, and easy to lead to a single point of failure, which is difficult to adapt to the large-scale data access; Secondly, because of the information management and maintenance involves a number of different departments which will lead to a lot of inconvenience for autonomous management.

Distributed information resource storage is an important development trend in the field of information sharing. At present, peer-to-peer computing system has been widely applied in file sharing, which confirms the scalability of distributed storage structure, but also produces some new problems. In unstructured peer-to-peer computing system based on flooding [7], information resources are scattered in various peer node which is easy to maintain, but leads to poor scalability. Besides, because resource discovery can only rely on flooding, search efficiency is low, and a lot of message load will lead to serious network congestion. The unstructured peer-to-peer computing system is only suitable for small range of information sharing. The structured peer-to-peer computing systems based on DHT [8] don't need information flooding in resources locating, which can be located directly to the destination node by using less routing information. The structured peer-to-peer computing systems have good extensibility and high search efficiency of information resources. But due to the complexity of algorithm and not supporting complex query conditions, the structured peer-to-peer computing systems are not suitable for information resources dynamic change scene and not conducive to autonomous management of resources.

Combined with efficiency and manageability of the centralized storage under the small scale condition and scalability of distributed storage, the Section designs a scalable distributed information resource storage model (SDIRSM) which is suitable for the application of data exchange under wide area network and is used to organize various 
nodes within the network to ensure the efficiency of information resources organization. As shown in Figure 1, the nodes in the network are given three roles such as information provider, information users and registry. From the viewpoint of network logic level, the SDIRSM can be divided into resource layer, common node layer and super node layer.

Resource layer covers the information resources for the purpose of sharing, transmission and exchange in data exchange. In order to be able to share the resources effectively, users must understand the relevant information of resources, namely resource metadata information. The information provider of the upper layer is responsible for the collection of metadata information.

Common node layer includes various information providers and users, collectively known as the client node. Information provider is responsible for collect information of resources from the resource layer, and provides the information for registry according to the unified information model. When need to query a resources, information user directly send a query request to the registry. After receiving the query results, the information user establishes the connection with the information provider, and then obtains the needed resources from the lower resources layer.

Super node layer brings together the nodes which have stronger network computing ability, collectively known as the registry. In this model, the registries located in different region are group into a tree structure. Each registry is responsible for managing the node of a region, maintains the information resources in this area, and forms an autonomous cluster together with the adjacent client end node. This way of organization is fully considering the composition of the real physical organization structure, and can ensure the autonomy of each region.

The difference between the distributed structure of SDIRSM and C/S structure [9] is that: in a C/S structure, all shared information resources must be stored in the server side, and the resource download must be provided by the server-side; In the model of SDIRSM, the specific resource contents are always stored on a local machine, and registry only maintains the metadata information related to resources. Because the SDIRSM has the advantages of hybrid peer-to-peer computing structure, which can effectively avoid the single point of failure problem, the SDIRSM has high expansibility and flexibility; at the same time, the design of super node in SDIRSM, to a certain extent, can improve the network load balance, high stability and efficiency.

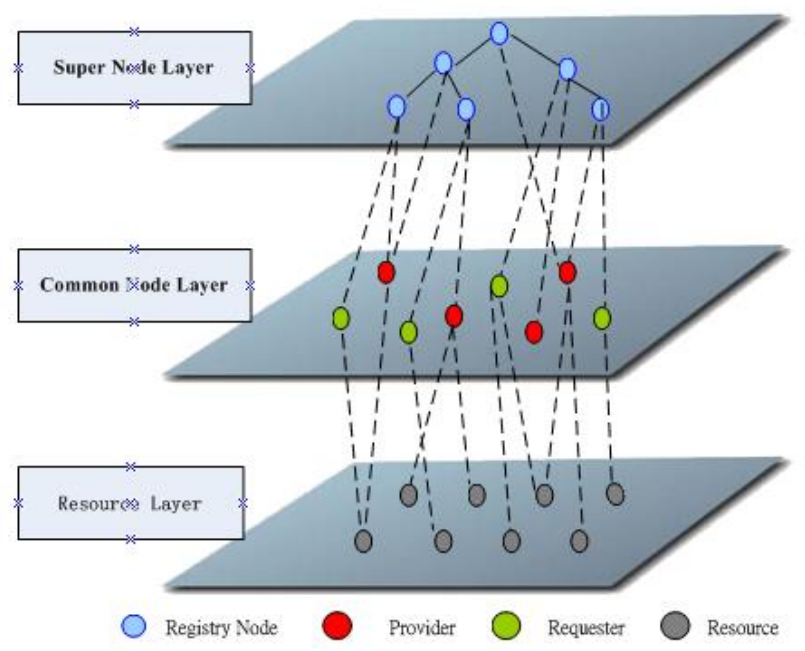

Figure 1. Scalable Distributed Information Resource Storage Model 


\section{Organization of the Registry}

In distributed information resource storage model, the nodes in data exchange network are divided into multiple regions, each region is manage and maintain by super node with strong calculating ability, the super node is known as the registry. In super node layer, the organization and management of the registry will become the key factors influencing the searching efficiency. In this paper, considering the efficient traversal algorithm of tree structure and the similarity between tree structure and organization structure in the application of E-Government, a tree storage model is adopted to organize the registry. The mechanism of join and exit of registry will mainly be discussed in the following Section.

System administrator is responsible for the configuration of the parent node information. Once the parent node is determined, the structure of the directory tree can be determined.

\subsection{Tree Model}

In reality, the physical network generally has tree characteristics. Tree structure is completely consistent with organization structure of many industries and applications, such as government agencies set up, personnel organization, etc. The nodes close to the location in space have the more probability that they need to find other information resources. At the same time, in computer science, a tree structure has a mature algorithm. We can use these algorithms for tree traversal and maintenance work. For information query, according to different requirements, query range can be flexibly controlled in this node, the subtree or the entire tree structure. Therefore, considering the efficient traversal algorithm of tree structure and the similarity of application organization structure in e-government, we intend to use the tree storage model to organize the registration center, which makes the user more convenient and efficient sharing and access to each other's information between these distributed registration center nodes, thus improving the overall efficiency of information sharing.

Considering autonomous regional registration center, registration center nodes distributed in different region can be organized into a tree hierarchical structure with reference to the physical organization. System administrators need to configure the parent node of information register center, so as to set up the father son relationship between the various registration centers. As shown in Figure 2, we through the stepwise selection at all levels of the registration center, the parent node configuration information of each node, so that the registration centers of all regions gradually are built into a complete distributed directory tree structure.

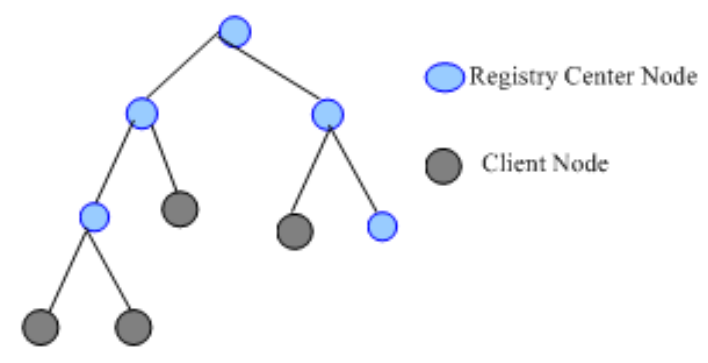

Figure 2. Registry Directory Tree

\subsection{The Join of a Registry}

The process of a registry joined to the directory tree is as follows:

(1)The registry reads the parent node information of local configuration services, through the interaction with the parent node, the join of a new node is completed. 
(2) If the parent node is not in the logical network, the node will attempt to establish connection with the parent node according to certain time interval. Once the parent node joins the network, the node can establish connection with the parent node.

(3) Once the parent node receives the join information of a child node, the information of the child node will be added to the corresponding data table.

Over time the registry will be able to dynamically access and update the activity list of child node, thus helping the registry decide to forward the search request to which child nodes in resources search.

\subsection{The Exit of a Registry}

In data exchange, generally the node with strong computing and processing capability can be regarded as a server, which is responsible for routing forwarding and regional management. These nodes are chosen as the registry. Assume that these nodes will not easily out of Web application system, therefore the paper does not consider the registry was forced to withdraw. If a problem occurs that a registry has exited but not known by other registry, which can be solved through sending test message. Once the message has not been sent successfully, the system will be the default that the registry exits, then client nodes in the region of the registry will no longer be involved in resource sharing.

\section{The Dynamic Register and Update of Information Resource Mechanism}

The register of information is just the first step for information use. Only the registered resource information can be used by the requestor. Information provider is responsible for register shared resource information in the specified registry. After resource information is registered, as the situation changes, resource information may need to be deleted or modified, that is information update. Hence it is very important to consider how to dynamically access the update information of resource so as to ensure the effectiveness of information and to avoid the change of resources cannot timely response to the registry.

From the above analysis, it can be seen that the constant change of the resource information may result in the information's dynamic registration, update, and delete, and so on. In order to guarantee the validity of the information resources, reduce the load caused by update messages, the dynamic register and update of information resource mechanism is designed as follows.

(1) According to certain time interval, the client periodically checks whether the resources information has been changed;

(2) If the resources to join, update, or exit, the client will automatically access the update information of resources, and publish the update information in the registry node in this region, to ensure that the latest information is maintained by the registry in this region.

(3)Once the registry receives update messages from the client, it will accordingly process depending on the message type and cache metadata information of update or register in the database.

Due to the registry maintains the global index information, so the change of the resource information will cause that index information will release update information along the path of leaf nodes to root node in the directory tree. Although compared with that all the registries should be keep in sync in complete copying strategies, this method has largely reduced the update message payload, but how to further reduce the redundant information and at the same time ensure the consistency of the information is still the issue to consider. The higher the registry level, the more the number of child nodes connected, which will lead to more frequently receive the index update information, which can lead to performance degradation. In order to improve the system performance, a non-uniform information distribution mechanism is adopted to dynamically update the index 
information [10-11]. When the registry node receives index update message from child node, it will not immediately forward the update message to its parent node, but filter these update message according to cache information, then will forward some filtered update message to its parent node at lower update frequency.

\section{Distributed Information Resource Search Algorithm}

Resource discovery and positioning is the basic function of data service center. For distributed information storage model, because resource information may be stored in a number of different registries, the client cannot know which node the information meeting the requirement is stored. So the process of resource discovery is to send the user's query request to the appropriate registry node and to find the right resources. The efficiency of resource discovery and positioning will seriously affect the performance of data service center. Therefore one of the key problems that must be solved is to use what kind of search strategy to quickly return the most appropriate resources.

The client will first submit query requests to the registry node in the region. The registry node is responsible for resource discovery of the super node level and eventually returns the appropriate resources. For the registry node, the process of resource discovery can be divided into two steps.

(1) The registry node queries information in the local directory server.

(2) The registry node searches resource between other registry nodes in the directory tree, that is to forward query request to other registry node and collect the query results.

\subsection{Local Information Query}

Local information query is mainly to implement query operation on the local directory server based on the qualification of query requests submitted by the client, and to obtain appropriate resources information which meet user requirement by the selection and match of resources.

At present, the main methods of resources are divided into the grammatical level match and the semantic level match. The grammatical level match refers to the simple match based on information name, such as UDDI [12]. The grammatical level match is relatively simple, but the precision is low and it is difficult to ensure the correctness. The semantic level match's resources description of the information is need to use ontology to solve the heterogeneity of traditional resource description, enhancement of the information resources and the behavior of semantic description. In the matching algorithm, because rely on the logical deduction and reasoning, the semantic level match has high precision but low efficiency, poor usability.

In view of the above question, we used a method of multidimensional resource selection, i.e., without introducing semantic context, to improve the accuracy of information query, to return to the most appropriate resource to the user as much as possible. Multidimensional resource selection is based on multiple attribute metadata in client released as matching parameters for resource selection. The query conditions composed of these attributes participated in resource matching are as shown in Figure 3. The <ResourceTitle> field cannot be empty. The composite field of $\langle$ Scope $\rangle$ represents the search scope used for the the query forwarding between the register center. The <MatchThreshold> and $<$ ResultsSize $>$ fields represent the maximum results match degree and find resources query set, used for resource selection. 


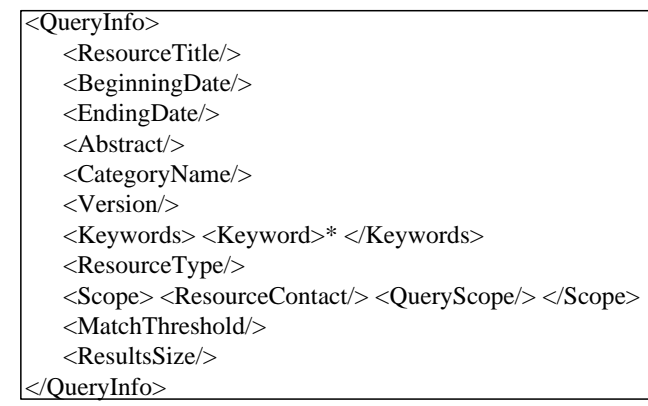

Figure 3. The Query Condition of Resources Match

\subsection{Distributed Information Query}

After completing the local information query, the registry node will further search resources in registry nodes of other areas, that is to forward query request to other registry node and collect the query results. Taking advantage of the characteristics of tree storage structure, the biased flooding algorithm based on index information is designed to implement distributed information resource search process. The core idea of the algorithm lies in that when the registry node forwards query request to the child node, the registry node doesn't use the random flooding method. According to the local cache information and query range, the registry node forwards query request to ensure that all the nodes forwarded can guide the positioning process according to the useful index information. Therefore, compared with the other random flooding algorithm, the algorithm is more effective. The flow chat of the algorithm is shown in Figure 4. According to different requirements, the registry node can flexibly control the scope of the query within the sub tree, other sub tree or whole tree. After forwarding query request, the registry node which starts a query request will be in a limited amount of time to collect the response information from the registry in other areas, transform the query results into a unified format, and finally return the results to the client node. At the same time, by taking the cache management, it makes the query first query local cache information in order to improve search efficiency.

In order to verify the validity of the biased flooding algorithm based on index information, the simulation experiment is designed. The experiment simulates the tree-like hierarchical structure of the registry node and resources distribution and query process. The biased flooding algorithm based on index information is compared with other random flooding algorithms under the tree structure. In the simulation experiments, the following two measurement parameters are designed.

1. The mean number of query forwarding: in a query, the number of registry nodes which the query request is forwarded to, namely forward hop, which is the main factor influencing the load under a tree structure.

2. Query hit ratio: the number of requests which can find effective and qualified resources in proportion to the number of all requests. 


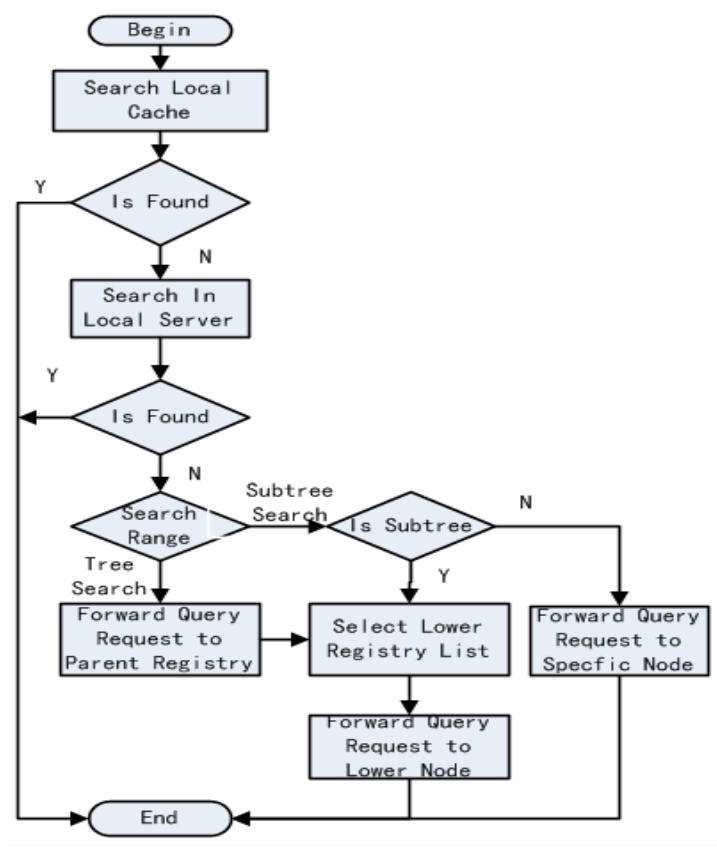

Figure 4. Distributed Information Resource Search Algorithm

Figure 5 depicts the influences that the three kind algorithms affect the mean number of query forwarding as the increase of the number of the registry node, which reflects the query message load. $\mathrm{M}$ is referring to matching degree of resources query. The experimental results show that with the increase of $\mathrm{m}$ value, the number of nodes forwarded using random flooding algorithms under the tree structure is large; the mean number of query forwarding using the biased flooding algorithm based on index information is little. At least the mean number of query forwarding using the biased flooding algorithm based on index information doesn't increase with the increase of the number of registry nodes, thus caused the less query message load.

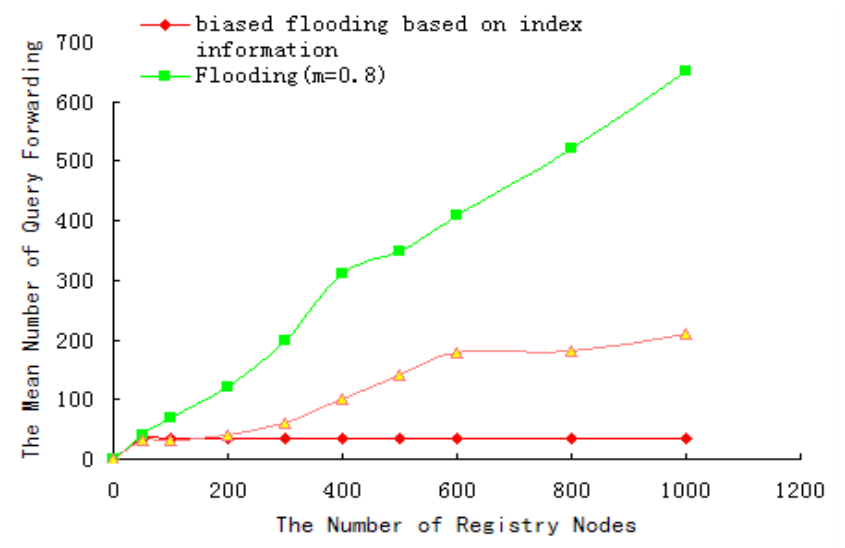

Figure 5. The Number of Registry Nodes and the Mean Number of Query Forwarding

Assuming that resource metadata information contains two attributes, query success shows that the query conditions in a query request match two related properties of the resources. Attr is referring to the number of attributes. It can be seen from the Figure 6 that, the Query hit ratio of random flooding algorithm is the lowest $(\mathrm{m}=0.5)$, and the Query hit ratio of biased flooding algorithm based on index information is the highest. 


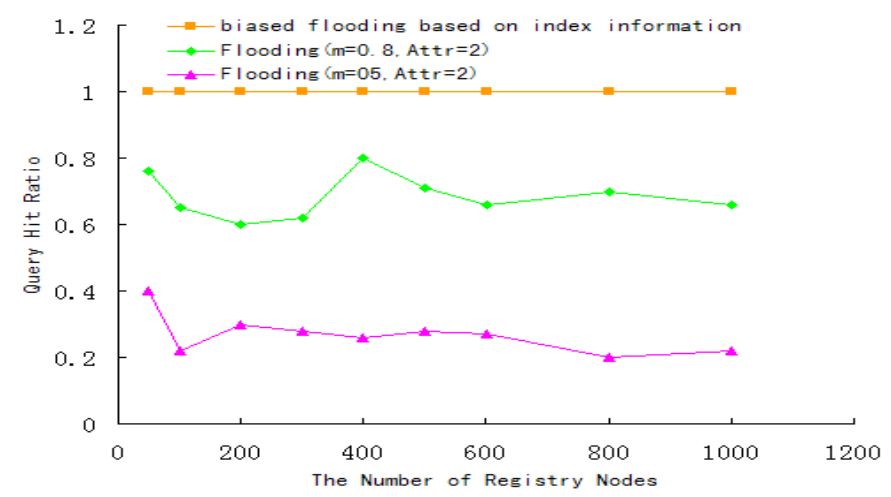

Figure 6. The Number of Registry Nodes and Query Hit Ratio

The experimental results show that:

(1) Due to the forwarding query requests without rely on useful information for forwarding, random flooding algorithm $(\mathrm{m}=0.5)$ causes the number of nodes forwarded is less in the query, but Query hit ratio is very low.

(2) Due to the use of the cache information and limit the query scope, the biased flooding algorithm based on index information both reduces the message payload by reducing query forwarding number and improves the Query hit ratio. The biased flooding algorithm based on index information is a very effective query algorithm.

\section{Conclusion}

In data exchange, data service should better describe, publish and discover resource information to provide effective information sharing service to users.

This paper has deeply studied the key problem facing in the field of data services, analyzed the extensibility, discovery efficiency and effectiveness of data service, designed a distributed information resource storage model, discussed the organization of the registry, proposed a new dynamic register and update of resource information mechanism, and designed a distributed information searching algorithm based on the distributed information resource storage model. Finally the simulation results are given. The experimental results show that the biased flooding algorithm based on index information can not only reduce the message payload by reducing query forwarding number and can improves the query hit ratio.

\section{Acknowledgment}

This work was funded by Science and Technology Project of Ministry of Housing and Urban-rural Development of the People's Republic of China (Grant No.2014-K8-057), Beijing Municipal Outstanding Talents Fund (Grant No. 2011D005017000002, Grant No. 2013D005017000017), Science and Technology Project of Beijing Municipal Commission of Education (Grant No. KM201410016002), Beijing Municipal Natural Science Foundation (Grant No. 4144072), Qianjiang Talents Project of Zhejiang Province (Grant No. 2011R10087), Scientific Research Foundation of Beijing University of Civil Engineering and Architecture (Grant No. 00331613002).

\section{References}

[1] J. Vilko and A. Rumpu, "Journal of System and Management Sciences", vol. 1, no. 5, (2011), pp. 106-114.

[2] C. L. Enrique, "Architecting the service oriented data center", 2007 IEEE International Conference on e-Business Engineering Workshop, (2007), pp. 694-700. 
[3] A. Motomitsu and H. Takuro, "A study on a resource allocation algorithm for on-demand data center services", 2008 International Conference on Advanced Communication Technology, vol. 1, (2008), pp. 295-300.

[4] G. Luca, R. G. Lo and G. Salvatore, “An adaptive routing mechanism for P2P resource discovery”, 2005 IEEE International Symposium on Cluster Computing and the Grid, vol. 1, (2005) May, pp. 205-212.

[5] C. Pablo and N. Leandro, "Collectives: A framework for self-adaptive P2P applications", Proceedings of the 6th Workshop on Adaptive and Reflective Middleware, (2007).

[6] M. Moreno, M. Matteo and O. Salvatore, "Resource discovery in a dynamic grid environment", Proceedings of the 16th International Workshop on Database and expert systems applications, (2005).

[7] M. Soohong, "Holliday JoAnne, Super-peer availability prediction strategy in unstructured P2P network", 6th International Conference on Network and Parallel Computing, (2009) October, pp. 23-29.

[8] Zhu Y. and Hu Y., "Efficient, proximity-aware load balancing for DHT-based P2P systems", IEEE Transactions on Parallel and Distributed Systems, (2005), pp. 349-361.

[9] W. Li and L. Peng, "Upgrade ERP from C/S to B/S based on Web service", 2005 International Conference on Services Systems and Services Management, vol. 1, (2005), pp. 593-597.

[10] V. Iyengar, S. Tilak, M. J. Lewis and N. B. Abu-Chazaleh, "Non-uniform information dissemination for dynamic grid resource discovery", Proceeding of the Third IEEE International symposium on network computing and applications, (2004).

[11] A. Husain, S. K. Makki, L. Osborne and B. Sun, "A highly adaptable information dissemination strategy", International Conference on Digital Enterprise and Information Systems, (2011).

[12] P. Roberto, "A lightweight inter-node operation for UDDI cloud", 2008 12th Enterprise Distributed Object Computing Conference Workshops, (2008).

\section{Authors}

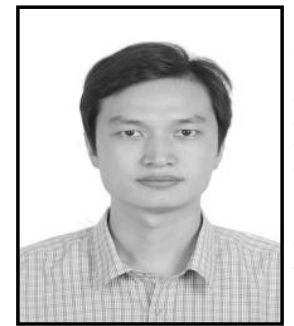

Xiaoxuan Ma, he received the $\mathrm{PhD}$ degrees from BeiHang University in 2010. Since 2010, he has been a lecturer at the School of Electrical and Information Engineering, Beijing University of Civil Engineering and Architecture. His research interests include data mining, distributed systems, distributed algorithms, adaptive prediction algorithms.

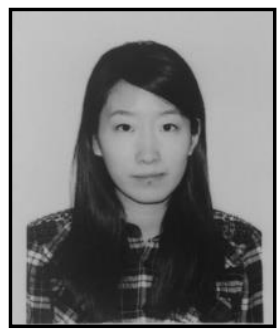

Yiping Huang, she was born in 1992. Since 2014 she has been a Graduate student of School of Electrical and Information Engineering, Beijing University of Civil Engineering and Architecture. Her research interests include building electrical and intelligent, distributed systems.

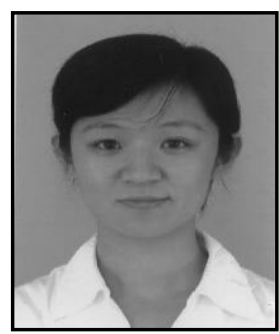

Junyan Yi, she received the B.S. degree and M.S. degree from Shandong University, Shandong, China in 2001 and 2005 respectively, and received the $\mathrm{PhD}$ degree from University of Toyama, Toyama, Japan in 2009, all in computer science. Between 2007 and 2009, she worked as a Research Assistant at University of Toyama. Between 2009 and 2012, she worked as an Associate Professor in the College of Computer Science \& Technology, Zhejiang University of Technology, China. She joined the Department of Computer Science \& Technology, Beijing University of Civil Engineering and Architecture, China in 2012 as an Associate Professor. Her main research interests include intellectual information technology, neural network, pattern recognition, image processing and optimizations problems. 